\title{
A Case of Pulmonary Tumor Thrombotic Microangiopathy Improved by the Treatment with Prednisolone
}

\author{
Yoshio Tomizawa, ${ }^{1}$ Masakiyo Yatomi, ${ }^{1}$ Masanori Iwashina, ${ }^{2}$ \\ Akihiro Yoshii, ${ }^{1}$ Reiko Yoshino, ${ }^{1}$ Kousuke Takei, ${ }^{1}$ \\ Tomohito Kuwako, ${ }^{1}$ Mai Tomizawa, ${ }^{1}$ Makoto Kondo ${ }^{3}$ \\ and Ryusei Saito ${ }^{1}$
}

\begin{abstract}
Pulmonary tumor thrombotic microangiopath (PTTM) presents with severe manifestations and rapid progression and is difficult to diagnose ante mortem. We report a case of gastric adenocarcinoma diagnosed with PTTM before death. Chest XR and CT showed ground-glass shadows in the bilateral lung fields, gastric adenocarcinoma was demonstrated by gastrointestinal fiber scopy and biopsy, and poorly differentiated carcinoma cells were detected in the pulmonary arterial blood by pulmonary wedge aspiration cytology on cardiac catheterization. After diagnosis of PTTM, prednisolone therapy of 30 $\mathrm{mg} /$ day was started. Dyspnea and hypoxemia were improved on the next day of administration of prednisolone. Although PTTM is rare, it should be considered the differential diagnosis of pulmonary hypertension and progressive respiratory failure with diffuse ground-glass shadow on chest radiographies. Furthermore, administration of prednisolone should be considered one of the treatment for the dyspnea by PTTM. (Kitakanto Med J 2013; 63:253 255)
\end{abstract}

Key words : gastric adenocarcinoma, pulmonary hypertension, pulmonary tumor thrombotic microangiopath, pulmonary wedge aspiration cytology, prednisolone

\section{Introduction}

Pulmonary tumor thrombotic microangiopath (PTTM) is characterized by widespread fibrocellular intimal proliferation of small pulmonary arteries and arterioles in patients with metastatic carcinoma. ${ }^{1}$ It presents with clinically severe manifestations and rapid progression and is difficult to diagnose ante mortem. Here, we report a case of PTTM diagnosed by pulmonary wedge aspiration cytology and improved by the treatment with prednisolone.

\section{Case Report}

A 73-years old woman was consulted the clinic, because of dry cough and dyspnea on exertion. Chest XR showed bilateral pleural effusions. Since common cold and congestive heart failure were suspected, antitussive drug and diuretic drug were administered. Although pleural effusions were decreased, dyspnea worsened and she was admitted to the hospital. Chest $\mathrm{XR}$ and CT showed ground-glass shadows in the bilateral lung fields and bilateral pleural effusions (Fig. 1). Cardiac ultrasonography showed enlargement of the right atrium and ventricle. Pulmonary arterial pressure was increased $66 / 23$ (mean 38 ) $\mathrm{mmHg}$ on cardiac catheter examination. Though beraprost sodium and warfarin potassium initiated for the treatment of pulmonary hypertension, the symptoms did not improve. Then, since class $\mathrm{V}$ poorly differentiated carcinoma cells (Fig. 2) were detected in pulmonary arterial blood by pulmonary wedge aspiration cytology on cardiac catheterization, the patient was transferred to National Nishigunma Hospital.

The patient's past history was unremarkable.

1 Department of Respiratory Medicine, National Nishigunma Hospital, 2854 Kanai, Shibukawa, Gunma 377-8511, Japan

2 Department of Pathology Medicine, National Nishigunma Hospital, 2854 Kanai, Shibukawa, Gunma 377-8511, Japan

3 Takase Clinic, 885-2 Minami-oorui, Takasaki, Gunma 370-0036, Japan

Received: May 16, 2013

Address: YOSHIO TOMIZAWA Department of Respiratory Medicine, National Nishigunma Hospital, 2854 Kanai, Shibukawa, Gunma 377-8511, Japan 

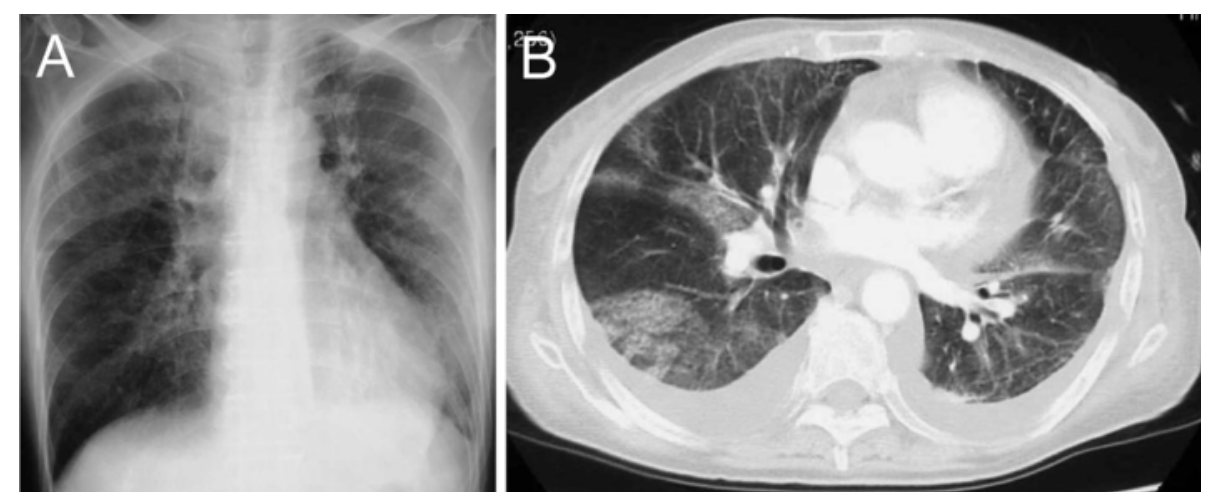

Fig. 1 Chest XR (A) and CT (B) showed ground-glass shadows in the bilateral lung fields and bilateral pleural effusion.

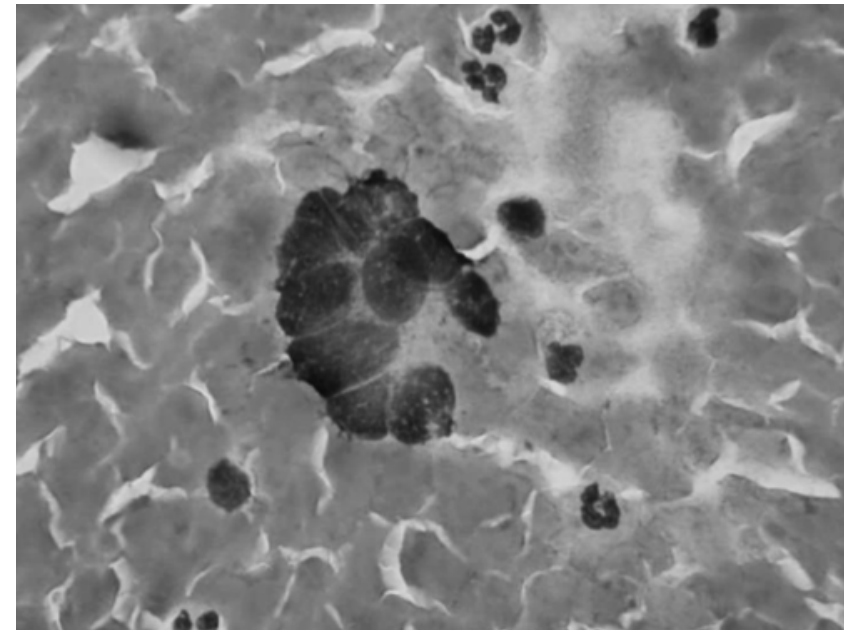

Fig. 2 Pulmonary arterial blood specimen by pulmonary wedge aspiration cytology showed poorly differentiated carcinoma cells with a high $\mathrm{N} / \mathrm{C}$ ratio (Papanicolaou stain $\mathrm{X} 400)$.

There was no history of smoking, and her family history was unremarkable. On examination, the patient's temperature was $37.2^{\circ} \mathrm{C}$. There was no cyanosis or clubbed fingers. There were no rales and no murmur. The abdomen was soft and flat without pain or tenderness. Neurological examination was unremarkable.

Laboratory findings were as follows: blood gas analysis on $\mathrm{O}_{2} 2 \mathrm{~L} / \mathrm{min}, \mathrm{pH} 7.471, \mathrm{pCO}_{2} 30.4 \mathrm{mmHg}$, $\mathrm{pO}_{2} 60.4 \mathrm{mmHg}, \quad \mathrm{HCO}_{3}-21.7 \mathrm{mmol} / \mathrm{L}, \quad \mathrm{A}-\mathrm{aDO}_{2} 89.4$, $\mathrm{SaO}_{2} 92.9 \%$. On hematological analyses, WCC 11 . $2 \times 10^{9} / \mathrm{L}, \mathrm{Hb} 109 \mathrm{~g} / \mathrm{L}$ and platelet count $286 \times 10^{9} / \mathrm{L}$. Biochemical analyses were unremarkable except for total protein $5.1 \mathrm{~g} / \mathrm{dL}, \quad$ BUN $27 \mathrm{mg} / \mathrm{dL}$ and CRP 2 . $64 \mathrm{mg} / \mathrm{dL}$. On serological analyses, there was no elevation of $\mathrm{SP}-\mathrm{A}, \mathrm{SP}-\mathrm{D}$ or KL6. Anti-nuclear antibody titer was slightly positive at $1: 40$, but tests for other autoantibodies such as rheumatoid factor or myeloperoxdase-antineutorophil antibody were negative. In tests for tumor markers, sialyl stage-specific antigen-1 was elevated to $69 \mathrm{U} / \mathrm{mL}$, but carcinoem- bryonic antigen, squamous cell carcinoma and pro gastrin-releasing peptide were negative.

Since the patient had tarry stool and vomited blood, gastrointestinal fiber scopy (GIF) was immediately performed. Type 2 gastric cancer on Borrman's classification of endoscopic findings was detected in an anterior wall of the corpus of the stomach, and poorly differentiated carcinoma was demonstrated on biopsy. We therefore diagnosed pulmonary tumor thrombotic microangiopathy (PTTM) from gastric cancer based on chest CT findings, GIF findings, pulmonary hypertension and pulmonary wedge aspiration cytology. Since respiratory failure was very severe, prednisolone therapy of $30 \mathrm{mg} /$ day was started. Dyspnea and hypoxemia were improved on the next day of administration of prednisolone. Amount of administration of the oxygen was reduced and general condition was recovered day after day. However, hypoxemia was rapidly progressed on 25 days after treatment by prednisolone, she died on the 34th hospital day due to respiratory failure.

At autopsy, poorly differentiated carcinoma of the stomach was detected and it invaded the peritoneum.

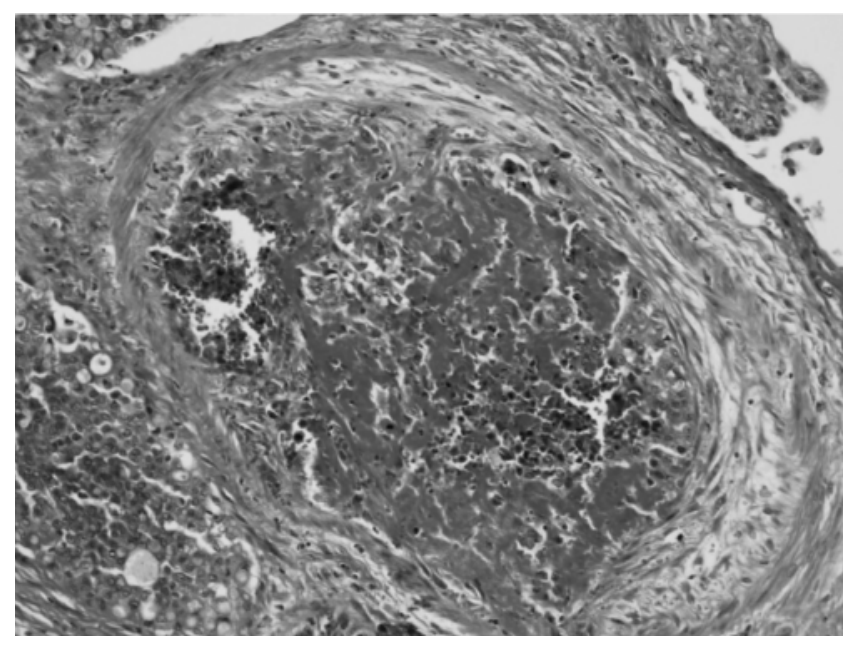

Fig. 3 Autopsy finding of the lung showed tumor embolisms and fibrocellular intimal proliferation of small pulmonary arteries. 
Tumor cells were also detected in from microcapillaries of small arteries, arterioles and lymph vessels in the lung. Obstruction and narrowing of the small arteries by fibrocellular intimal proriferation and fibrotic thickness around the vessels (Fig. 3).

\section{Discussion}

Pulmonary tumor thrombotic microangiopath (PTTM) is characterized by widespread fibrocellular intimal proliferation of small pulmonary arteries and arterioles in patients with metastatic carcinoma. ${ }^{1}$ The tumor most commonly associated with PTTM is adenocarcinoma, and most frequent primary site is the stomach. In the present case, PTTM was also associated with poorly differentiated adenocarcinoma of the stomach. This condition presents with clinically severe manifestations such as pulmonary hypertension and right-side heart failure. ${ }^{2}$ Open lung biopsy or transbronchial lung biopsy are necessary for the definitive diagnosis of PTTM. However, since the clinical course is very rapid, many patients die before the diagnosis is established. Though it was reported that pulmonary wedge aspiration cytology is available for the diagnosis of pulmonary tumor embolism, ${ }^{3,4}$ few cases have been reported. In the present case, tumor cells were detected by pulmonary wedge aspiration cytology during the course of examining pulmonary hypertension. Then, gastric cancer was demonstrated by GIF and biopsy. Therefore, the present patient was a rare case in which PTTM from gastric adenocarcinoma was diagnosed before death.

Chest CT finding of PTTM was reported that there was no massive embolism in pulmonary artery and multiple small nodules and/or multiple granular shadows were spreaded in the lung fields. ${ }^{5}$ Pathological features of PTTM were tumor cells metastasized to the pulmonary vascular system at microscopic level, deposition of platelet and fibrin microthrombi, fibrocellular subinitimal proliferation and smooth muscle colonization of the lesional complex. ${ }^{6}$ In the present study, chest CT showed ground-glass shadows in the bilateral lung fields. Although PTTM is very rare, it should be considered in the differential diagnosis of pulmonary hypertension and progressive respiratory failure with diffuse ground-glass shadow on chest XR and/or CT.

Treatment of the PTTM was very difficult, because the clinical course was very rapid and the performance status was very poor due to the complication of severe dyspnea in most patients. In the present case, administration of prednisolone was effective for improvement of dyspnea of PTTM. Therefore, prednisolone therapy should be considered one of the treatment for the dyspnea by PTTM.

\section{References}

1. von Herbay A, Illes A, Waldherr R, et al. Pulmonary tumor thrombotic microangiopathy with pulmonary hypertension. Cancer 1990; 66 : 587-592.

2. Bassiri A, Haghighi B, Doyle R, et al. Pulmonary tumor embolism. Am J Respir Crit Care Med 1997 ; 155 : 20892095.

3. Bhuvaneswaran J, Venkitachalam C, Sandhyamani S. Pulmonary wedge aspiration cytology in the dagnosis of recurrent tumour embolism causing pulmonary arterial hypertension. Int J Cardiology 1993 ; 39: 209-212.

4. Masson R, Ruggieri J. Pulmonary microvascular cytology. A new diagnostic application of the pulmonary artery catheter. Chest 1985; 88: 908-914.

5. Nishikata R, Terashima M, Soeta $N$, et al. A case of gastric cancer suspected pulmonary tumor thrombotic microangiopathy. Jpn J Gastroenterol Surg 2005 ; 38 : 13061311.

6. Pinckard JK, Wick MR. Tumor-related thrombotic pulmonary microangiopathy : review of pathologic finding and pathophysiologic mechanisma. Ann Diagn Pathol 2000; 4: 154-157. 